\title{
CAMBIOS EN LA HETEROCEE NEIDAD DEL BOSQUE NATIVO EN LA PATACONIA ANDINA DE ARGENTINA Y SU IMPACTO SOBRE LA BIODIVERSIDAD EN LOS SECTORES DE BORDE
}

\section{Francisco Carabelli, Manuel Jaramillo* y Silvio Antequera}

CENTRO DE INVESTIGACIÓN Y EXTENSIÓN FORESTAL ANDINO-PATAGÓNICO (CIEFAP)

ARGENTINA

*Dirección actual: CIBIO. Universidad de Alicante

\section{INTRODUCCIÓN}

La región andino patagónica ampara uno de los ecosistemas boscosos más singulares del planeta, que constituye una de las últimas fronteras forestales en su tipo (Bryant et al., 1997). Estos bosques son particularmente abundantes en endemismos y particularidades adaptativas (Donoso, 1995), al tiempo que cubren las cuencas altas y medias - $y$ aún los valles- de los ríos de la región (Rusch, 1989). A pesar de la baja densidad demográfica $-1,5$ hab./ $\mathrm{km}^{2}$ ), las áreas boscosas disminuyen desde fines del siglo XIX y principios del siglo XX a causa de alteraciones extensivas de origen humano, tales como explotaciones ganaderas, incendios y extracciones forestales, para mencionar a las más significativas (Carabelli y Antequera 2003). Estudios recientes han corroborado este proceso al com- probar una reducción de la superficie original de bosque, debido a la predominancia de los cambios fundamentalmente "negativos" provocados por el hombre (Carabelli et al., 2002).

El ciprés de la cordillera (Austrocedrus chilensis D. Don Pic. Serm et Bizzarri) es una de las especies arbóreas nativas que ha sido más severamente afectada por la influencia humana, al punto que desde 1997 se halla incluida en la Lista Roja de Especies Amenazadas de la Unión Internacional para la Conservación de la Naturaleza (Farjon et al., 1998). El desarrollo no planificado de asentamientos humanos que ha incrementado dramáticamente el riesgo de incendios, el cambio de uso para habilitar tierras para el pastoreo de ganado ovino y bovino, el reemplazo por especies exóticas de más rápido crecimiento, el desarrollo agrí́cola en pequeñas pero numerosas propiedades y la explotación 
intensiva para el empleo de la madera han reducido, en algunos casos drásticamente, la superficie original.

Desde una perspectiva ecológica, estos impactos alteran negativamente el hábitat original, al aislar y reducir el área del mismo y producir un aumento proporcional de bordes con relación a las condiciones de interior. Distintos autores (Franklin y Forman 1987; Van Dorp y Opdam 1987) señalan que los cambios en la forma y conectividad de los parches provocan alteraciones en la riqueza de especies, en su distribución y en la probabilidad de que se manifiesten perturbaciones necesarias para los procesos de sucesión. De acuerdo con Murcia (1995) el aumento en la cantidad de ambientes de borde produce efectos a nivel de los componentes abióticos del ecosistema — cambios microclimáticos- y bióticos directos e indirectos — cambios de la abundancia de especies y en los procesos de interacción.

En este contexto, hemos abordado el estudio de los cambios en la heterogeneidad de los bosques nativos de ciprés de la cordillera en dos niveles: el primero de ellos es el de paisaje, esencialmente cuantitativo, para determinar las alteraciones en superficie que han afectado a los distintos tipos forestales e identificar la naturaleza de algunas de tales alteraciones. El restante nivel, de caracter cuali-cuantitativo involucra una escala predial, para analizar la biodiversidad asociada a tres sectores de borde adyacentes a matrices originadas por incendios forestales, plantaciones de Pinus ponderosa y talas rasas.

\section{MATERIAL Y MÉTODOS}

El área de estudio se ubica en el NO de la provincia de Chubut, Argentina ( $42^{\circ} 09^{\prime} 25.7^{\prime \prime} \mathrm{S}$ $\left.71^{\circ} 23^{\prime} 08.5^{\prime \prime} \mathrm{O}\right)$ y posee una superficie de 7000 ha. Se realizó un análisis detallado de elementos del paisaje sobre un fotomosaico ensamblado con 25 fotografías aéreas en infrarrojo tomadas en el año 1970 a una escala aproximada 1:20000 (Fig. 1). La identificación de zonas incendiadas y plantaciones de especies exóticas establecidas en el periodo 1970 -2001 se realizó sobre una imagen satelital IKONOS multiespectral obtenida en enero de 2001 ( 4 bandas, resolución espacial de 4 x 4 m). Para la ortorrectificación de las fotografías y la imagen se confeccionó un Modelo Digital de Terreno (MDT) a partir de curvas de nivel (equidistancia $100 \mathrm{~m}$ ) digitalizadas de cartas topográficas del Instituto Geográfico Militar (IGM). Para la elaboración del MDT, la ortorrectificación de la imagen y las fotos y el ensamble del fotomosaico se utilizaron diferentes módulos de los programas Erdas Imagine 8.4 de la empresa ERDAS y ArcView 3.2a de la empresa ESRI.

Sobre el fotomosaico del año 1970 se delimitaron elementos del paisaje (Fig. 2) definidos para este estudio como parches de bosque según la siguiente clasificación: Tipo forestal: 1) Ciprés puro, 2) Ciprés-Coibue (cobertura de ciprés $>50$ \%), 3) Coihue-Ciprés (cobertura de coihue > 50\%), 4) Ciprés-Matorral con las siguientes especies arbóreas o arbustivas acompañantes: ñire (Nothofagus antarctica), laura (Schinus patagonicus) y radal (Lomatia hirsuta). Para los 4 tipos forestales se separaron parches según las siguientes clases de densidad: 1) Denso (distancia entre dominantes < $5 \mathrm{~m}), 2$ ) Semidenso (distancia entre 5 y $10 \mathrm{~m}$ ), 3) Ralo (distancia > $10 \mathrm{~m}$ ). Se estableció una Mínima Unidad Cartografiable (MUC) de $2500 \mathrm{~m}^{2}$, habiéndose individualizado sólo elementos de superficie mayor a la misma. Sobre la imagen IKONOS se delimitaron las áreas incendiadas y las plantaciones (Fig. 3), únicas alteraciones consideradas en esta presentación. Para ambas actividades se realizaron chequeos de terreno. El cálculo de superficies por clases y el análisis de los cambios ocasionados por las alteraciones consideradas se realizó con la extensión Xtools del programa ArcView.

En los sectores de borde analizamos sólo la diversidad de especies leñosas —árboles, arbustos y enredaderas-, menores a $5 \mathrm{~cm}$ de diámetro a la altura del pecho, debido a la imposibilidad de tomar en cuenta todos los componentes bióticos de una zona determinada, pues se tendría que incluir desde los microorganismos hasta los organismos de mayor talla (Halffter 1998; Montes Pérez 2002). Entre los sitios analizados, el borde lindante con un área quemada corresponde a un 
bosque denso de ciprés de aproximadamente 25 metros de altura media, con exposición noreste. El incendio tiene una antigüedad de 16 años y no se han realizado medidas de mitigación, por el contrario, se constató la presencia de ganado vacuno. Mientras tanto, en la zona de borde con exposición norte adyacente a la tala rasa realizada hace 15 años (Fig. 4), los árboles de ciprés alcanzan 20 metros de altura. Finalmente, el sector lindero a la plantación de pinos esta expuesto hacia el noroeste, con árboles de hasta $30 \mathrm{~m}$ de altura. La plantación de $P$. ponderosa tiene 25 años de edad y carece de manejo silvícola.

Se analizó la alfa diversidad por medio de la riqueza de especies $(S)$ presente en cada ambiente. Debido a la diferencia en la cantidad de individuos identificados en cada sitio, principalmente entre el "borde quemado" (BQ) con "borde tala rasa" (BTR) y "borde plantación de pinos" (BPP) se ensayaron curvas de rarefacción con el programa Ecosim 7.0. Se ensayaron estimadores no parametricos con el programa StimateS 6.0 (Colwell 2000), entre ellos Chao ${ }^{1}$, $\mathrm{Chao}^{2}$, Jacknife de primer orden $\left(\mathrm{Jack}^{1}\right)$ y de segundo orden $\left(\mathrm{Jack}^{2}\right)$. La diversidad beta o diversidad entre hábitat se evaluó mediante índices cualitativos (Índice de Sokal y Sneath), cuantitativos (Coeficiente de similitud de Sørensen) e índices de reemplazo de especies (Índice de Magurran). La diversidad gamma se analizó con base en los componentes alfa, beta y la dimensión espacial (Schluter y Ricklefs 1993; fide Moreno 2001).

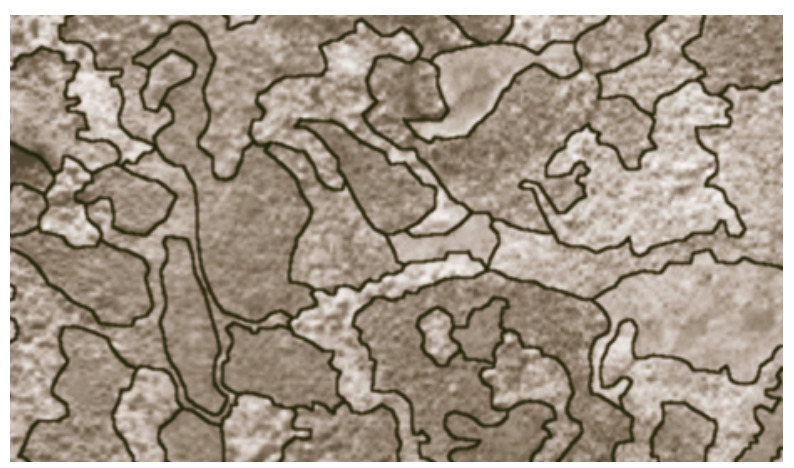

Figura 1. Distribución original del bosque nativo en 1970 sobre un fotomosaico en escala 1:20000.

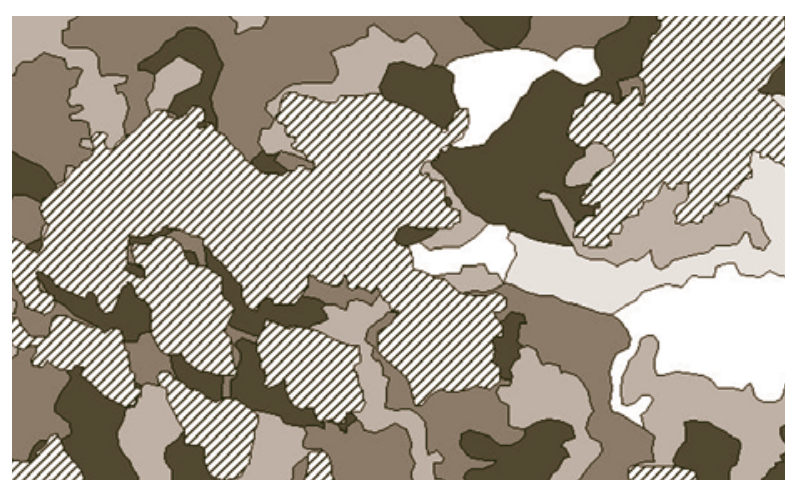

$\mathrm{Ci}_{\mathrm{Ci}-\mathrm{Co}} \square \mathrm{Co}_{\mathrm{C}-\mathrm{Ci}} \square$ Ci-Mat. $\square$ s/b VIII Plantaciones

Figura 2. Clasificación del bosque nativo por tipos forestales sobre el fotomosaico de 1970.

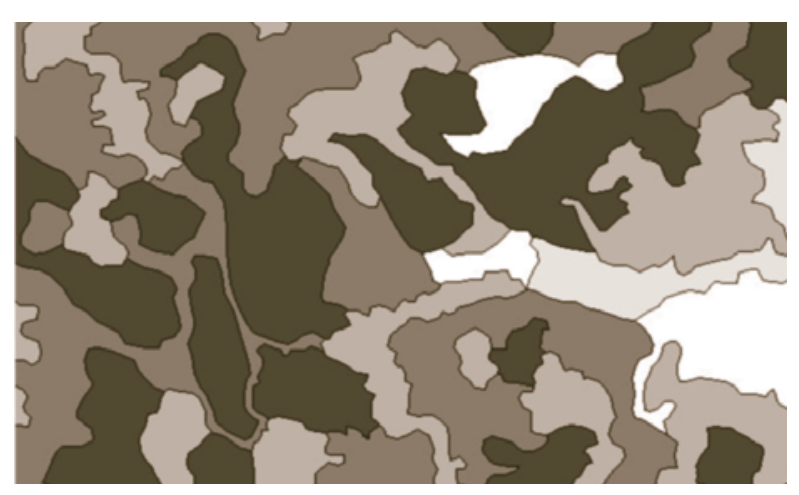

Ci $\square$ Ci-Co $\square$ Co.Ci $\square$ Ci-Matorral $\quad \square$ sin bosque

Figura 3. Reemplazo del bosque nativo por plantaciones con especies exóticas ocurrido entre 1970 y 2001.

\section{RESULTADOS}

De acuerdo al análisis de los parches identificados sobre el fotomosaico del año 1970, el número total de polígonos pertenecientes a los cuatro tipos forestales ascendía a 1191 cubriendo una superficie de 4166 ha $-60 \%$ del área de estudio aproximadamente- - Asumiendo que no se produjeron cambios sustanciales en la densidad de los tipos forestales identificados y que las causas principales de modificaciones de la heterogeneidad del paisaje se debieron a la ocurrencia de incendios y al establecimiento de plantaciones, se evaluó que en el año 2001 el número de polígonos fue de 1572, 


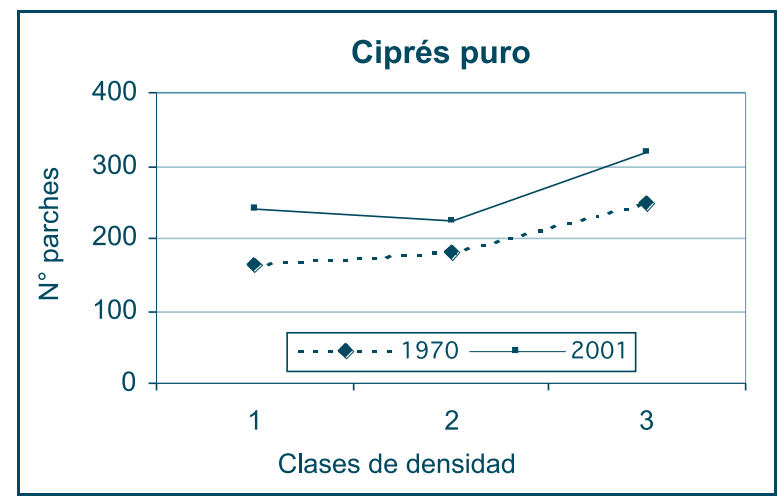

Diagrama 1. Variación en el número de parches de ciprés puro entre 1970 y 2001 para las tres clases de densidad consideradas

cubriendo un área de 3580 hectáreas. Ello puso de manifiesto que la heterogeneidad del paisaje forestal se intensificó negativamente al fragmentarse la superficie de bosque nativo a una tasa promedio de $32 \%$, cambio que se ejemplifica para el tipo forestal ciprés puro (Diagrama 1). Mientras tanto, la reducción total de superficie fue de $14 \%$.

El número total de parches afectado por el incendio ocurrido en 1987 asciende a 39 mientras que la superficie que ellos cubrían en el año 1970 era de 145 hectáreas (Tabla 1). Los tipos forestales más afectados fueron ciprés y ciprés-matorral, en particular para las clases de menor densidad. Ello obedece a que el incendio se produjo sobre una ladera de suelos pedregosos poco desarrollados con afloramientos rocosos, en general cubierta por bosques secos y matorrales bajos. Sobre la superficie incendiada se establecieron aproximadamente doce hectáreas de plantaciones, ocupando principalmente los sitios más húmedos que luego del incendio se cubrieron con un matorral de especies como el palo piche (Fabiana imbricata) y el espino negro (Colletia spinosisima).

Tabla 1.

Número de parches y superficie afectada por incendio

\begin{tabular}{lcccccc}
\hline \multirow{2}{*}{ Tipo forestal } & \multicolumn{5}{c}{ Clase de densidad } \\
\cline { 2 - 7 } & \multicolumn{2}{c}{1} & \multicolumn{2}{c}{2} & \multicolumn{2}{c}{3} \\
\cline { 2 - 7 } & NoP Sup. & NoP & Sup. & NoP & Sup. \\
\hline Ciprés puro & - & - & 8 & 21 & 17 & 66 \\
Ciprés-coihue & 2 & 5 & 3 & 5 & - & - \\
Coihue-ciprés & 1 & 1 & 1 & 4 & - & - \\
Ciprés matorral & - & - & 3 & 13 & 4 & 29 \\
\hline
\end{tabular}

La superficie total afectada por el reemplazo con plantaciones, considerando los cuatro tipos forestales y las tres densidades, asciende a 442 hectáreas (Tabla 2). Como puede apreciarse, los bosques densos fueron los que sufrieron el mayor impacto - 82 hectáreas - y dentro de ellos, los bosques de ciprés puro — 69 hectáreas—.

\section{Tabla 2}

Superficie de bosque nativo que fue reemplazada por plantaciones durante el periodo 1970 - 2001

\begin{tabular}{llll}
\hline Tipo forestal & \multicolumn{3}{c}{ Clase de densidad } \\
\cline { 2 - 4 } & 1 & 2 & 3 \\
\hline Ciprés puro 69 & 40 & 118 & \\
Ciprés-coihue & 48 & 10 & 7 \\
Coihue-ciprés & 28 & 1 & 1 \\
Ciprés matorral & 37 & 47 & 36 \\
\hline
\end{tabular}

Respecto al impacto que estas alteraciones causaron en los sectores de borde, la alfa diversidad, indicada como riqueza de especies, mostró diferencias entre los bordes analizados (Tabla 3).

\section{Tabla 3}

Riqueza de especies (S) y frecuencia total de individuos relevados en los tres hábitat estudiados.

\begin{tabular}{lcc}
\hline Hábitat & $S$ & Frecuencia \\
\hline Borde con plantación & 24 & 2066 \\
Borde con quemado & 16 & 4134 \\
Borde con tala rasa & 14 & 2313 \\
\hline
\end{tabular}

El ensayo con curvas de rarefacción permitió ratificar la mayor biodiversidad del BPP, mientras que evidenció que la riqueza de especies entre BTR y BQ no presenta diferencias significativas. Otros índices de diversidad alfa mostraron un comportamiento similar.

$\mathrm{Chao}^{2}$ fue el estimador que más se aproximó a la riqueza observada. Según este índice en cada sitio se han observado entre el 88 y el $96 \%$ de las especies esperadas. Cuando se analizó la biodiversidad alfa de los tres hábitat agrupados, el índice Jack ${ }^{2}$ proporcionó el mejor ajuste, indicando que las especies observadas representan un $90 \%$ de las 
esperadas. El estimador $\mathrm{Chao}^{1}$, como indicador de la estructura de la diversidad alfa, indicó que la riqueza de especies observada se encuentra entre el 89 y el $99 \%$ de las esperadas.

Los resultados de los diferentes indicadores de diversidad beta sugieren una amplia similitud entre BQ y BTR y una mayor divergencia entre estos hábitat con respecto a BPP. El índice de Sokal y Sneath tiende a suavizar estas diferencias. El índice cuantitativo de Sørensen, contrariamente a los cualitativos, muestra una mayor diferencia entre BTR y BPP que entre BQ y BPP, lo que podría estar relacionado con el alto número de individuos medido en BQ. El índice de Magurran se ha comportado como sugiere Moreno (2001), proporcionando valores de diversidad beta mayores cuando el número de especies entre los sitios comparados aumenta y también cuando éstos se vuelven más diferentes. La diversidad beta promedio fue 0,56 . De las 30 especies observadas en conjunto, sólo 11 se encuentran presentes en los 3 sitios.

La diversidad gamma o diversidad del paisaje fue de 28,89 especies, valor muy aproximado a las 30 especies identificadas en el conjunto de los tres hábitat analizados.

La mayor diversidad del BPP puede estar asociada a un microclima más favorable y al mayor impacto antrópico, que ha favorecido el establecimiento de algunas especies exóticas como rosa mosqueta (Rosa eglanteria), Pino ponderosa y pino oregón (Pseudotsuga menziessii), que no se encuentran presentes en los demás sitios.

De estos resultados se desprende que el sitio de mayor importancia para la conservación es el BPP debido a la mayor riqueza de especies nativas, al grado más elevado de amenaza por las actividades antrópicas y a la necesidad de gestión de las especies introducidas, especialmente las señaladas precedentemente, cuya regeneración altamente agresiva representa una amenaza para el mantenimiento de la biodiversidad actual. En segundo nivel de importancia se encuentra el BQ, no sólo por el numero de especies, sino porque presenta un mayor número de individuos de especies raras.

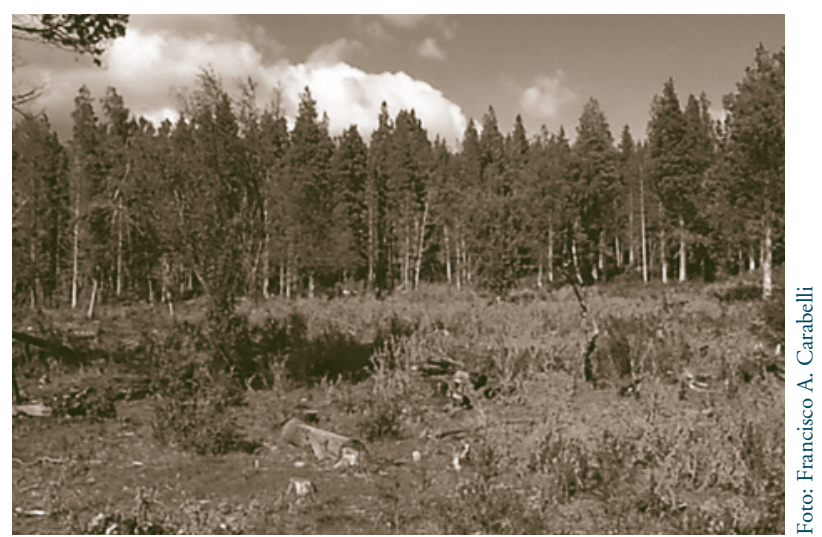

Figura 4. Borde de bosque de ciprés lindante con un sector de tala rasa.

\section{BIBLIOGRAFÍA}

BRYANT, D., D. NIELSEN y L. TANGLEY. 1997. Las últimas fronteras forestales. Ecosistemas y economías en el límite. Instituto de Recursos Mundiales. 42 p.

CARABELLI, F., R. SCOZ, H. CLAVERIE, M. JARAMILLO y M. GÓMEZ. 2002. Detection and assessment of positive and negative changes on the heterogeneity of forest landscapes: fragmentation analysis of "ciprés de la cordillera" forests in Patagonia Andina, Argentina. International Conference "Disturbed landscapes: Analysis, Modeling and Valuation”. Cottbus, Alemania. 107-108.

CARABELLI, F. y S. ANTEQUERA. 2003. La reducción de la superficie de bosques en la región andino patagónica: análisis de algunos factores involucrados. Reporter Emergency News Agency. $10 \mathrm{p}$.

COLWELL, R. K. 2000. EstimateS: Statistical estimation of species richness and shared species from samples. Version 6.0b1. User's Guide and application (http://viceroy.eeb.uconn.edu/estimates).

DONOSO, C. 1995. Estado actual y posibilidades de manejo del bosque nativo andino-patagónico. IV Jornadas Forestales Patagónicas. San Martín de los Andes.

FARJON, A. 1998. Data collection forms for conifer species completed by the IUCN/SSC Coni- 
fer Specialist Group between 1996 and 1998.

FRANKLIN, J. F. y R.T.T. FORMAN. 1987. Creating landscape patterns by forest cutting: ecological consequences and principles. Landscape Ecology (1): 5-18.

HALFFTER, G. 1998. A strategy for measuring landscape biodiversity. Biology International, 36: 3-17.

MONTES PÉREZ, R. C. 2002. Potencialidades de los sistemas silvopastoriles para la generación de servicios ambientales. Conferencia electrónica (http://lead-es.virtualcentre.org/es/ele/conferencia3 /comenta1_3.htm).

MORENO, C. E. 2001. Métodos para medir la biodiversidad. M\&T-Manuales y Tesis SEA,
Vol. 1. Zaragoza, 84 p.

MURCIA, C. 1995. Edge effects in fragmented forests: Implications for conservation. Trends in Ecology and Evolution.

RUSCH, V. 1989. Determinación de las trancisiones de estado en bosques de lenga (Nothofagus pumilio). CONICET, Informe Final de Beca de Perfeccionamiento. 75 p.

VAN DORP, D. y P.F.M. OPDAM. 1987. Effects of patch size, isolation and regional abundance on forest bird communities. Landscape Ecology (1): 59-73. 